\title{
Lipopolysaccharide induces pyroptosis through regulation of autophagy in cardiomyocytes
}

\author{
You-Fu He $\mathrm{He}^{1,2,3 \#}$, Jing Huang ${ }^{1,2,3}$, Yu Qian ${ }^{4}$, De-Bin Liu ${ }^{5}$, Qi-Fang Liu ${ }^{1,2}$ \\ ${ }^{1}$ Department of Cardiology, Guizhou Provincial People's Hospital, Guiyang, China; ${ }^{2}$ Guizhou Provincial Cardiovascular Disease Clinical Medicine \\ Research Center, Guiyang, China; ${ }^{3}$ Medical College, Guizhou University, Guiyang, China; ${ }^{4}$ Department of Cardiology, The Second Affiliated \\ Hospital of Zunyi Medical University, Zunyi, China; ${ }^{5}$ Department of Cardiology, Shantou Second People's Hospital, Shantou, China \\ Contributions: (I) Conception and design: YF He, QF Liu; (II) Administrative support: QF Liu; (III) Provision of study materials or patients: DB Liu; \\ (IV) Collection and assembly of data: YF He, J Huang; (V) Data analysis and interpretation: YF He, Y Qian; (VI) Manuscript writing: All authors; (VII) \\ Final approval of manuscript: All authors. \\ "These authors contributed equally to this work. \\ Correspondence to: Dr. Qi-Fang Liu. Department of Cardiology, Guizhou Provincial People's Hospital, No. 83, Zhongshan East Road; Guizhou \\ Provincial Cardiovascular Disease Clinical Medicine Research Center, Guiyang 550000, China. Email: liuqiff66@163.com.
}

Background: Autophagy, a stress response in eukaryotic cells, is closely related to cardiogenic diseases. Pyroptosis, a newly discovered way of programmed cell death, also plays an important role in cardiovascular disease. However, the role and relationship of autophagy and pyroptosis in lipopolysaccharide (LPS)-induced inflammatory response of cardiomyocytes were still unclear.

Methods: Western blot was performed to determine the expression of poly ADP-ribosepolmesera-1 (PARP-1), LC3B, NLRP3 and GSDMD in cardiomyocytes after the treatment of LPS. Transfection of si-LC3B, western blot and immunofluorescence (IF) staining were performed to investigate the role of autophagy in LPS-induced pyroptosis. Co-immunoprecipitation (Co-IP) assays and quantitative realtime PCR (qRT-PCR) were conducted to explore whether PARP-1 binding to LC3B and modulating its expression. Transfections of si-PARP-1, western blot and IF were carried out to confirm the role of PARP-1 in the regulation of LPS-induced pyroptosis by autophagy.

Results: LPS induces autophagy and pyroptosis in cardiomyocytes, enhanced the level of autophagy and inhibited the level of pyroptosis in the concentration of $4 \mu \mathrm{g} / \mathrm{mL}$. We further proved that autophagy inhibits LPS-induced pyroptosis in cardiomyocytes. In addition, PARP-1 binding to LC3B and regulate the expression of LC3B. Finally, we proved that knockdown of PARP-1 rescued the inhibition of autophagy on LPS-induced pyroptosis of cardiomyocytes.

Conclusions: LPS induces pyroptosis through regulation of autophagy via PARP-1 at a specific concentration, above which it causes deposition of autophagy flow to promote pyroptosis. Inhibiting LPSinduced pyroptosis could be a promising therapeutic target in treating cardiovascular diseases.

Keywords: Lipopolysaccharide (LPS); autophagy; pyroptosis; poly ADP-ribosepolmesera-1 (PARP-1); cardiomyocytes

Submitted May 06, 2021. Accepted for publication Aug 30, 2021.

doi: $10.21037 / \mathrm{cdt}-21-293$

View this article at: https://dx.doi.org/10.21037/cdt-21-293

\section{Introduction}

Autophagy, as a stress response in eukaryotic cells, involves various signaling pathways and the degradation and circulation of biological macromolecules and damaged organelles in cells (1-4). In the process of autophagy, cells degrade damaged organelles, incorrectly folded proteins and other substances in the cytoplasm into fatty acids, amino acids and other substances by using hydrolases in lysosomes, 
and then recycle and reuse these substances to maintain the stability of substances and energy in the cells (5-8). LC3B, Beclin-1 and p62 are the signature proteins of autophagy (9-12). Cardiogenic diseases are considered to be associated with autophagy (13-15). In the stress response, activation of autophagy started the degradation of various macromolecules and organelles, which promote the survival of cardiomyocytes (16-18). However, the autophagy mechanism caused by severe stress can lead to self-destruction, eventually leading to heart failure (19).

Pyroptosis is a newly discovered way of programmed cell death, which is different from cell apoptosis and cell death, which is accompanied by inflammatory reaction. There are two different pathways which causing pyroptosis: a canonical pathway depending on the activation of caspase-1 (CASP1) and a non-canonical pathway depending on the activation of caspase-4/5/11 (CASP4/5/11) (20-22). Recent studies have found that pyroptosis plays an important role in cardiovascular disease $(22,23)$. Therefore, further study of the role of pyroptosis in the occurrence and development of cardiovascular diseases will be of great significance for the prevention and treatment of diseases. In addition, pyroptosis has been found to be associated with autophagy (24-28). For instance, hypothermia has been reported to alleviate cerebral ischemic injury by autophagy activation and pyroptosis suppression (29). Prostatic hyperplasia has been reported to resulted by protein peroxiredoxin 3 according to inhibiting autophagy and activating pyroptosis (30). Therefore, pyroptosis may be regulated via autophagy in cardiovascular disease.

Poly ADP-ribosepolmesera-1 (PARP-1) is a posttranslational modification enzyme existed in most eukaryotic cells (31). Under normal conditions, PARP-1 activity is very low, and PARP-1 activity is increased during DNA damage, catalyzing poly ADP ribosylation of receptor proteins and participating in DNA repair (32). On the other hand, excessive activation of PARP-1 induced by oxidative stress leads to rapid consumption of $\mathrm{NAD}+$ and further consumption of ATP, resulting in cell dysfunction and necrosis $(33,34)$. PARP-1 has the function of keeping chromosome structure intact, participating in DNA replication and transcription, and playing a role in maintaining genomic stability and cell death $(31,35,36)$. Studies have shown that PARP-1 plays an important role in neuronal apoptosis, cardiovascular disease, cerebral ischemiareperfusion injury, neurodegeneration and other diseases $(31,32,37,38)$. For instance, PARP-1 has been reported to promote autophagy in cardiomyocytes by regulating Foxo3a (37). PARP-1 has also been found to inhibited attenuates cardiac fibrosis resulted from myocardial infarction via modulating autophagy (38). In addition, GCBI database query results show that PARP-1 may be a transcript of LC3B. Therefore, PARP-1 may participate in lipopolysaccharide (LPS) induced inflammatory response of cardiomyocytes via regulating autophagy.

Based on the above results, we hypothesized that LPS induces pyroptosis by regulating cardiomyocyte autophagy. According to our study, LPS induces autophagy and pyroptosis in cardiomyocytes, enhanced the level of autophagy and inhibited the level of pyroptosis in the concentration of $4 \mu \mathrm{g} / \mathrm{mL}$. Knockdown of LC3B, we proved that autophagy inhibits LPS induced pyroptosis in cardiomyocytes. Further experiments showed that PARP-1 binding to LC3B and regulate the expression of $\mathrm{LC} 3 \mathrm{~B}$. In addition, knockdown of PARP-1 rescued the inhibition of autophagy on LPSinduced pyroptosis of cardiomyocytes. To sum up, we draw the conclusion that LPS induces pyroptosis through regulation of autophagy via PARP-1 in cardiomyocytes. We clarified the role of LPS and PARP-1 regulating autophagy and pyroptosis in the occurrence and development of inflammatory process of cardiomyocytes, which is of great significance for the prevention and treatment of cardiovascular diseases.

We present the following article in accordance with the MDAR reporting checklist (available at https://dx.doi. org/10.21037/cdt-21-293).

\section{Methods}

\section{Cell isolation and culture}

The myocardial tissues of newborn mice were purchased from Hunan Fenghui Biotechnology Co., Ltd. (Changsha, China). Myocardial tissues of newborn mice were first digested by collagen II (CLS004176, Worthington, OH, USA) and trypsin (E7885, Sigma, St. Louis, MO, USA). Then the high purity cardiomyocytes were acquired by utilizing a discontinuous Percoll gradient centrifugation (P4937, Sigma, St. Louis, MO, USA). Cardiomyocytes were cultured in Dulbecco's modified Eagle's medium (DMEM) with $10 \%$ fetal bovine serum (FBS, GIBICO, 16000044, Thermo Fisher Scientific, Waltham, MA, USA) under the condition at $37{ }^{\circ} \mathrm{C}$ with $5 \% \mathrm{CO}_{2}$ in the humidified incubators. LPS (L4391) was purchased from Sigma Chemical Co (St. Louis, MO, USA). 


\section{Cell transfection}

si-LC3B, si-PARP-1, si-NC, ov-PARP-1 and ov-NC were designed by GenePharma (Shanghai, China). Transfections of cardiomyocytes were performed by Lipofectamine 2000 (Thermo Fisher Scientific, Waltham, MA, USA) following the manufacturer's instructions.

\section{RNA isolation and quantitative real-time PCR (qRT-PCR)}

Total RNAs of cardiomyocytes were first extracted by using TRIzol reagent (Sigma, St. Louis, MO, USA) according to the protocol of manufacturer. For qRT-PCR, cDNAs were first synthesized by utilizing SuperScript III reverse transcriptase kit (Sigma, St. Louis, MO, USA). Then, PCR were conducted by Taq-Man Universal PCR master mix (Sigma, St. Louis, MO, USA). Experiments of cDNAs synthesize and PCR were performed following the manufacturer's instructions. Relative expression of PARP-1 and LC3B mRNA were calculated by normalizing to $\beta$-actin. ABI 7500 real-time PCR system fluorescent dye method was performed for qRT-PCR. The fold changes were calculated with $2^{-\Delta \Delta C t}$ method. Primer sequences were showed as following: PARP-1, F: 5'-CTC TCC CAG AAC AAG GAC GAA G-3', R: 5'-CCG CTT TCA CTT CCT CCA TCT TC-3'. LC3B, F: 5'-TTA TAG AGC GAT ACA AGG GGG AG-3'; R: 5'-CGC CGT CTG ATT ATC TTG ATG AG-3'. $\beta$-actin, $F$ : 5'-CAT TGC TGA CAG GAT GCA GAA GG-3', R: 5'-TGC TGG AAG GTG GAC AGT GAG G-3'.

\section{Western blot}

Western blot was carried out to determine the protein expression of PARP-1, LC3B-I/II, NLRP3 and GSDMD. Samples were first prepared by utilizing ReadyPrepTM Protein Extraction Kit (Bio-Rad, Shanghai, China) following the instructions of manufacturer. Then, protein samples were separated by SDS-PAGE and transferred to PVDF membranes (Millipore, Germany). For immunoblotting, blots contained target proteins were treated with corresponding primary antibodies overnight at $4{ }^{\circ} \mathrm{C}$ and corresponding horseradish peroxidase-conjugated anti-rabbit second antibodies (611-035-215, 1:5,000, Jackson ImmunoResearch, West Grove, PA, USA) for $1 \mathrm{~h}$ at $37^{\circ} \mathrm{C}$. The primary antibodies (all from Abcam, Cambridge,
UK) used in this study were as follows: anti-PARP-1 (ab110915, 1:1,000), anti-LC3B (ab51520, 1:3,000), antiNLRP3 (ab214185, 1:1,000), anti-GSDMD (ab219800, 1:1,000), anti-caspase-1 (ab138483, 1:1,000) and anti- $\beta$-actin (ab6276, 1:5,000). Blots were viewed under the Odyssey Infrared Imaging System (LI-COR, Lincoln, NE, USA) and quantified by Image $J$ software (National Institutes of Health, Bethesda, MD, USA). The relative protein level was normalized to $\beta$-actin.

\section{Immunofluorescence (IF) staining}

Cardiomyocytes were first placed on glass slides at an appropriate density and then fixed with $4 \%$ paraformaldehyde for $15 \mathrm{~min}$. After that, cells were permeabilized by $0.1 \%$ Triton (dissolved in PBS) at room temperature for $30 \mathrm{~min}$. Then cells were treated by blocking solution, incubated by primary antibody overnight and hybridized by corresponding secondary antibody. The nucleus of cells was stained by DAPI. Finally, sections were visualized by a fluorescence microscope (Olympus, Japan).

\section{Co-immunoprecipitation (Co-IP) assay}

Firstly, preparation of samples was conducted. The samples were treated by IP lysis buffer. Protein samples were quantified by using Pierce ${ }^{\mathrm{TM}}$ BCA Protein Assay Kit (Cat\# 23225, Thermo Fisher, Waltham, MA, USA) following the instructions of manufacturer. For each sample, $1 \mathrm{mg}$ protein lysates were used for the experiment of IP. Secondly, antibody capture was conducted. Samples were incubated with IgG and anti-PARP-1. Next, lysates were incubated for $4 \mathrm{~h}$ at $4{ }^{\circ} \mathrm{C}$. Then the lysates were added with $25 \mu \mathrm{L}$ Protein A/G agarose. After the mix of lysates and Protein A/G agarose, the mixture was incubated for $2 \mathrm{~h}$ at $4^{\circ} \mathrm{C}$. Thirdly, washing was conducted by IP wash buffer. Finally, western blot was performed to immunoblot PARP-1 and LC3B.

\section{Statistical analysis}

All the data were analyzed by GraphPad Prism 7 (GraphPad Software, Inc., San Diego, CA, USA). All the data were shown as mean \pm standard deviation $(\mathrm{SD})$. Differences were confirmed by Student's $t$-test or one-way ANOVA. $\mathrm{P}<0.05$ was considered as a significant difference. All experiments were repeated three times independently. 

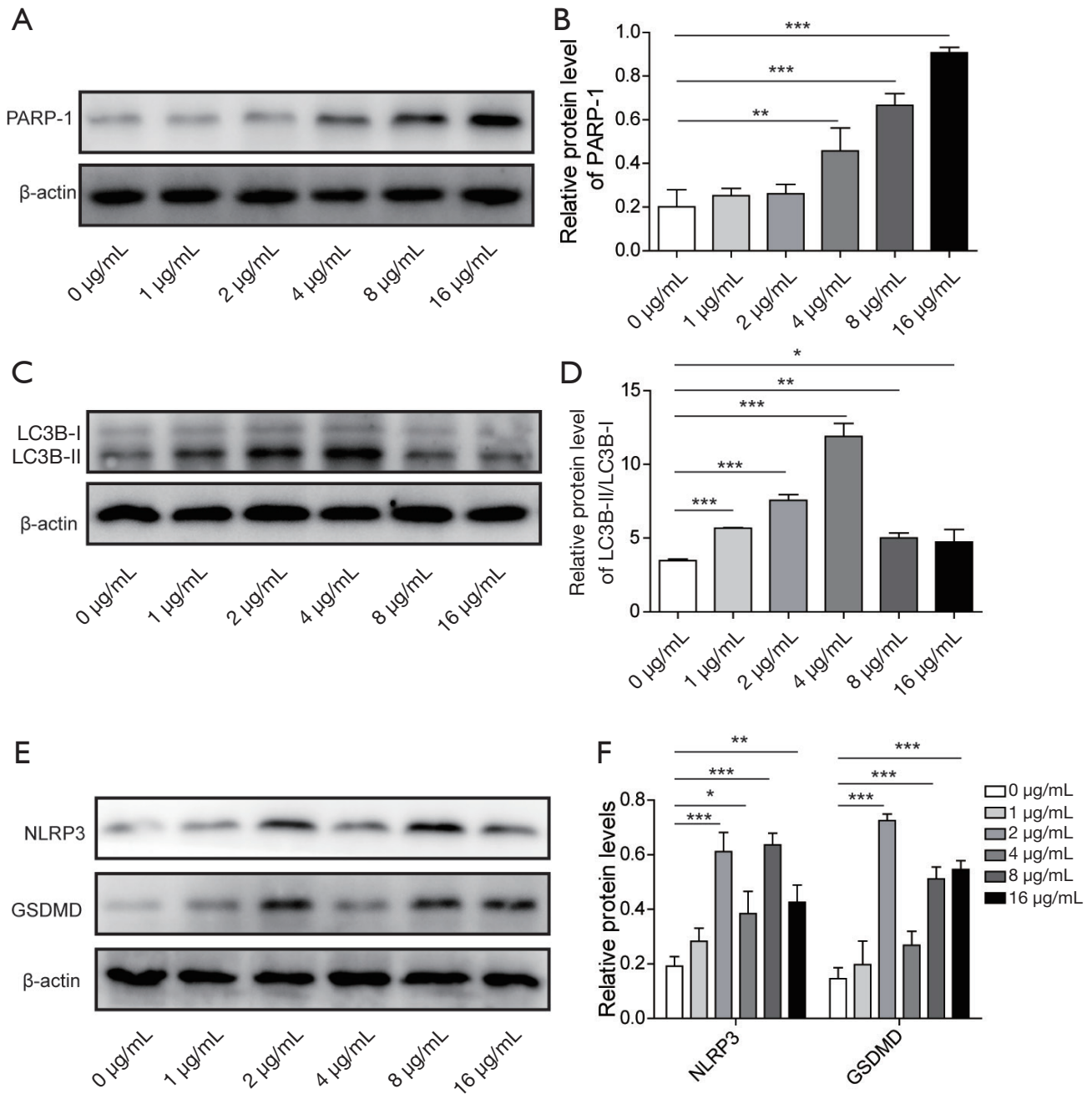

Figure 1 LPS induces autophagy and pyroptosis of cardiomyocytes. Cardiomyocytes were treated with LPS of different concentrations for $2 \mathrm{~h}$. (A,B) PARP-1 expression was quantified by western blot. (C,D) Expression of LC3B-I and LC3B-II were determined by western blot. (E,F) Expression of NLRP3 and GSDMD were determined by western blot. * $\mathrm{P}<0.05$; **, $\mathrm{P}<0.01$; ***, $\mathrm{P}<0.001$. PARP-1, poly ADPribosepolmesera-1; LPS, lipopolysaccharide.

\section{Results}

\section{LPS induces autophagy and pyroptosis of cardiomyocytes}

To determine the effect of LPS on autophagy and pyroptosis of cardiomyocytes, expression of PARP-1, LC3B-I/II, NLRP3 and GSDMD were determined by western blot in cardiomyocytes after treated with LPS in different concentrations. Cardiomyocytes were treated with LPS of different concentrations for $2 \mathrm{~h}$, and the results of western blot show that higher concentration of LPS enhanced the expression of PARP-1, indicating that LPS promotes the PARP-1 expression in a dose dependent manner (Figure $1 \mathrm{~A}, 1 \mathrm{~B})$. Then western blots were performed to quantify the expression of LC3B-I and LC3B-II, results show that LPS enhanced the ratio of LC3B-II and LC3B-I within the concentration of 0 to $4 \mu \mathrm{g} / \mathrm{mL}$, and decreased the ratio within the concentration of 4 to $16 \mu \mathrm{g} / \mathrm{mL}$, indicating that LPS promotes the autophagy within a certain concentration range, and decreases autophagy in higher concentrations (Figure 1C,1D).

The expression of NLRP3, cleaved caspase-1 and GSDMD were then quantify by performing western blot, results showed that expression of NLRP3, cleaved caspase-1 and GSDMD treated with LPS in the concentration of $4 \mu \mathrm{g} / \mathrm{mL}$ were lower than 2 and $8 \mu \mathrm{g} / \mathrm{mL}$, indicating that pyroptosis of cardiomyocytes were inhibited by LPS in the 
concentration of $4 \mu \mathrm{g} / \mathrm{mL}$ (Figure $1 E, 1 F$, Figure S1). To sum up, the results of western blot indicated that LPS induces autophagy and pyroptosis of cardiomyocytes. LPS enhanced the level of autophagy and inhibited the level of pyroptosis in the concentration of $4 \mu \mathrm{g} / \mathrm{mL}$.

\section{Autophagy inbibits LPS-induced pyroptosis of cardiomyocytes}

To explore the effect of autophagy on LPS-induced pyroptosis, expression of NLRP3 and GSDMD was determined by western blot and IF in cardiomyocytes treated with LPS and si-LC3B. qRT-PCR was first performed to measure the effect of transfection after the transfection si-LC3B. Results show that LC3BII mRNA expression was decreased by the transfection of si-LC3B compared with si-NC (Figure 2A). Western blot was then conducted, and showed that LC3B-II expression was decreased by the transfection of si-LC3B (Figure 2B). Expressions of LC3B-II, NLRP3, cleaved caspase- 1 and GSDMD were determined by western blot in cardiomyocytes after treated with LPS and si-LC3B. Compared with control group, the treatment of $4 \mu \mathrm{g} /$ $\mathrm{mL}$ LPS enhanced the expression of LC3B-II, NLRP3, cleaved caspase- 1 and GSDMD. However, the treatment of LPS and si-LC3B decreased the expression of LC3B-II and continue enhanced the expression of NLRP3, cleaved caspase- 1 and GSDMD, indicating that inhibition of autophagy promotes LPS-induced pyroptosis (Figure 2C, Figure S2).

Expression of NLRP3 and GSDMD was further determined by IF. Results showed that $4 \mu \mathrm{g} / \mathrm{mL}$ LPS enhanced NLRP3 expression, and knockdown of LC3B continue increased NLRP3 expression (Figure 2D,2E). Consistently, $4 \mu \mathrm{g} / \mathrm{mL}$ LPS enhanced GSDMD expression, and knockdown of LC3B continue increased GSDMD expression (Figure 2F,2G). In conclusion, autophagy inhibits LPS-induced pyroptosis in cardiomyocytes.

\section{$P A R P-1$ bind to $L C 3 B$ and regulate the expression of $L C 3 B$}

To investigate whether PARP-1 binding to LC3B and regulate the expression of LC3B, Co-IP was first performed to confirm the interaction of PARP1 and LC3B, then qRT-PCR and western blot was performed to quantify the expression of LC3B mRNA and protein after the overexpression of PARP-1. As shown in Figure 3A, PARP-1 and LC3B expressed in both of the input group and anti-PARP-1 group of the IP group, indicating that PARP-1 interact with LC3B. qRT-PCR was then performed in cardiomyocytes after transfected with ov-PARP-1, results showed that overexpression of PARP-1 enhanced relative expression of LC3B mRNA (Figure 3B). Consistently, western blot showed that overexpression of PARP-1 enhanced relative expression of LC3B protein (Figure 3C,3D). To sum up, these findings indicated that PARP-1 binding to $\mathrm{LC} 3 \mathrm{~B}$ and regulate the expression of LC3B.

\section{Inbibiting PARP-1 rescued the inbibition of autophagy on LPS-induced pyroptosis of cardiomyocytes}

To confirm the role of PARP-1 in the regulation of LPSinduced pyroptosis, expression of NLRP3 and GSDMD was determined by western blot and IF in cardiomyocytes treated with LPS and si-PARP-1. qRT-PCR was first performed to measure the effect of transfection after the transfection si-PARP-1. Results show that PARP-1 mRNA expression was decreased by the transfection of si-PARP-1 compared with si-NC (Figure 4A). Western blot was then conducted, and showed that PARP-1 expression was decreased by the transfection of si-PARP-1 (Figure 4B). Expression of LC3B-II, NLRP3, cleaved caspase-1 and GSDMD was determined by western blot in cardiomyocytes after treated with LPS and si-PARP-1. Compared with control group, the treatment of si-PARP-1 decreased the expression of LC3B-II and enhanced the expression of NLRP3, cleaved caspase-1 and GSDMD, indicating that knockdown of PARP-1 rescued the inhibition of autophagy on LPS-induced pyroptosis of cardiomyocytes (Figure 4C, Figure S3).

We further determined the expression of NLRP3 and GSDMD by performing IF. Results showed that knockdown of PARP-1 enhanced the percentage of NLRP3 positive cells (Figure 4D,4E). Consistently, knockdown of PARP-1 enhanced the percentage of GSDMD positive cells (Figure $4 F, 4 G$ ). In conclusion, knockdown of PARP-1 decreased the expression of LC3B-II, and enhanced the expression of NLRP3 and GSDMD. These findings reveal that knockdown of PARP-1 rescued the inhibition of autophagy on LPS-induced pyroptosis of cardiomyocytes.

\section{Discussion}

Autophagy, involves the degradation and circulation of 

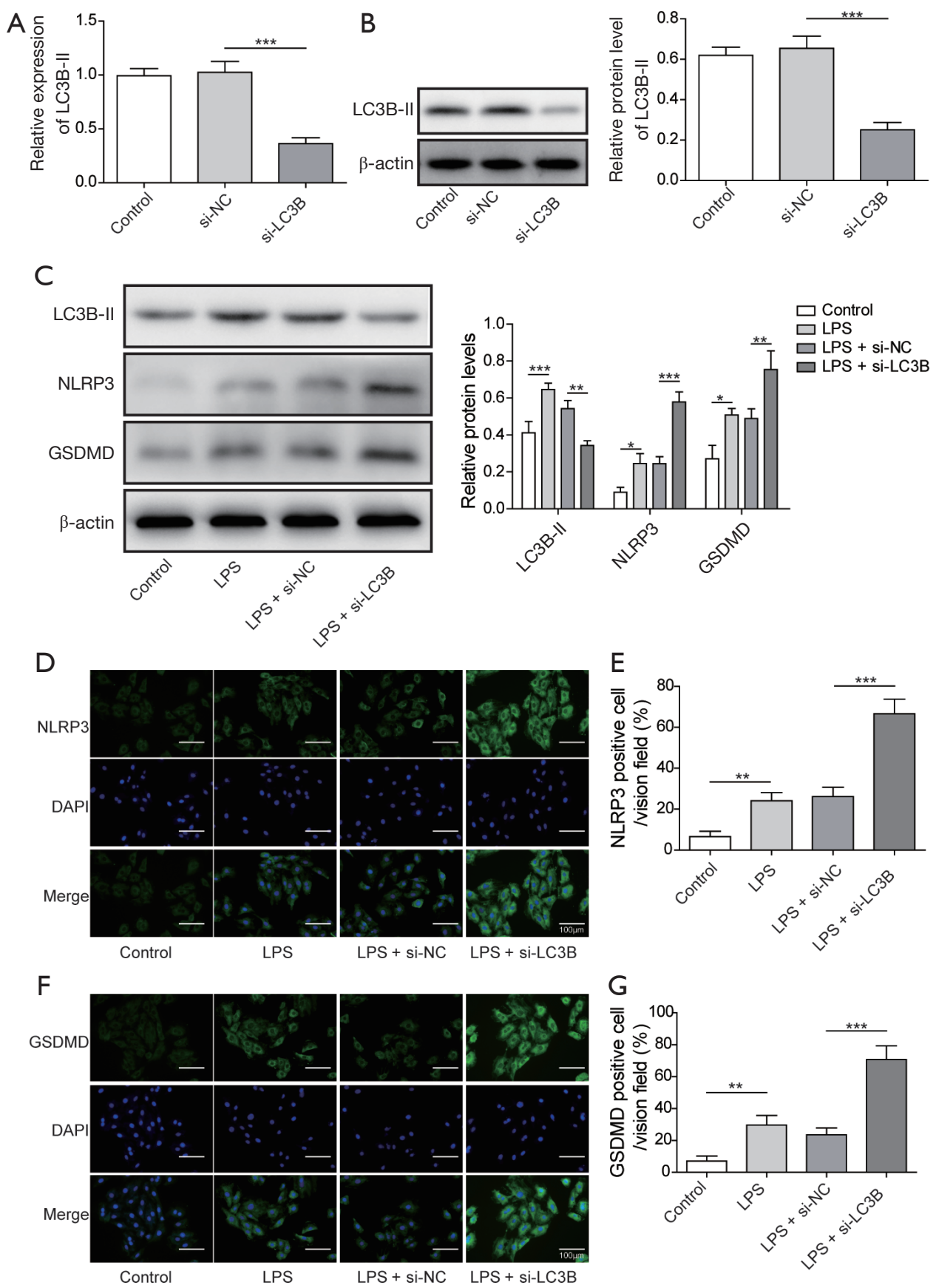

Figure 2 Autophagy inhibits LPS-induced pyroptosis of cardiomyocytes. Cardiomyocytes were transfected with si-LC3B and si-NC. (A) LC3B-II mRNA expression was detected by performing qRT-PCR. (B) LC3B-II protein expression was measured by western blot. (C) Western blots were performed to determine the expression of LC3B-II, NLRP3 and GSDMD in cardiomyocytes after transfected and treated with LPS of $4 \mu \mathrm{g} / \mathrm{mL}$. (D,E) Expression of NLRP3 in cardiomyocytes were detected by IF staining after transfected and treated with LPS of $4 \mu \mathrm{g} / \mathrm{mL}$. (F,G) Expression of GSDMD in cardiomyocytes were detected by IF staining after transfected and treated with LPS of $4 \mu \mathrm{g} / \mathrm{mL}$. *, $\mathrm{P}<0.05$; **, $\mathrm{P}<0.01$; ***, $\mathrm{P}<0.001$. LPS, lipopolysaccharide; qRT-PCR, quantitative real-time PCR; IF, immunofluorescence. 

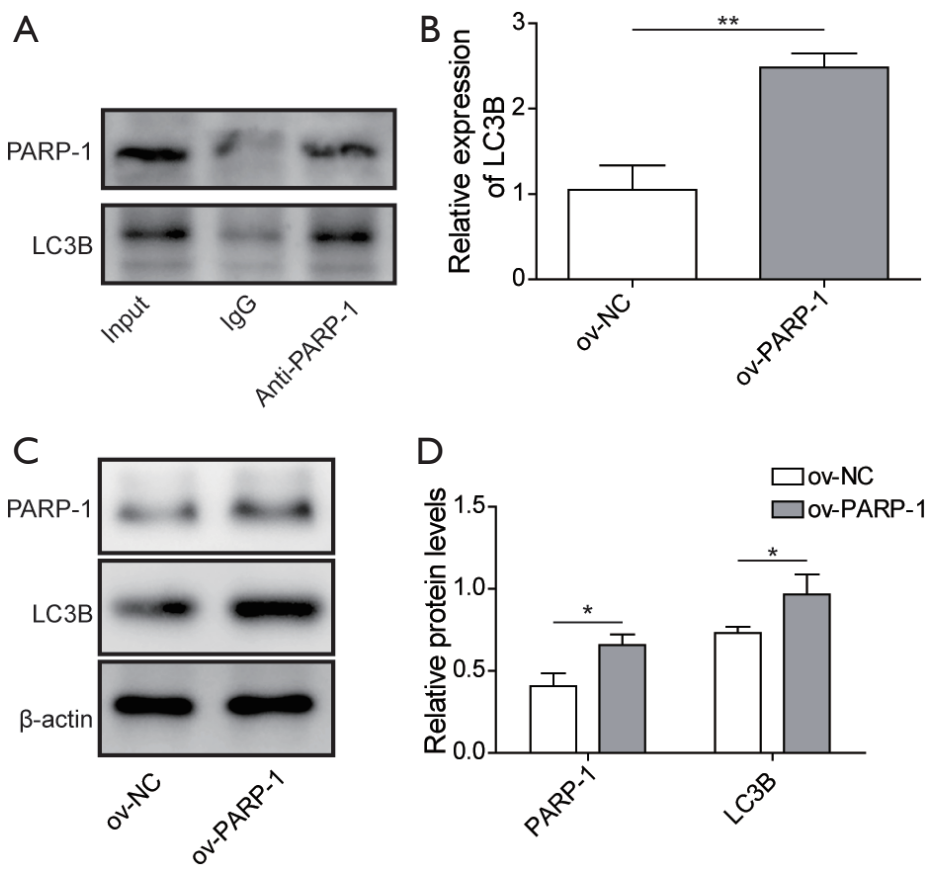

Figure 3 PARP-1 bind to LC3B and regulate the expression of LC3B. (A) The interaction of PARP-1 and LC3B was tested by Co-IP assays in cardiomyocytes. (B) The expression of LC3B was detected by qRT-PCR. (C,D) The expression of PARP-1 and LC3B were detected by western blot. *, $\mathrm{P}<0.05$; **, $\mathrm{P}<0.01$. PARP-1, poly ADP-ribosepolmesera-1; Co-IP, co-immunoprecipitation; qRT-PCR, quantitative realtime PCR.

biological macromolecules and damaged organelles in cells, is closely related to inflammation and cardiogenic diseases, and changes in the level of autophagy affect the degree of disease response (13-15). In the stress response, activation of autophagy triggers the degradation of various macromolecules and organelles, which promotes the survival of cardiomyocytes (16-18). Minocycline has also been reported to induce the autophagy of cardiomyocyte to inhibit sepsis-induced cardiac dysfunction via Akt/mTOR signaling pathway (16). However, the autophagy mechanism caused by severe stress can lead to self-destruction, eventually leading to heart failure (19). Therefore, to investigate the role of autophagy in LPS-induced inflammatory response of cardiomyocytes, we explored the role of LPS in autophagy and pyroptosis. According to our study, we found that LPS induces autophagy and pyroptosis of cardiomyocytes, enhanced the level of autophagy and inhibited the level of pyroptosis in the concentration of $4 \mu \mathrm{g} / \mathrm{mL}$. Furthermore, we found that autophagy inhibits LPS-induced pyroptosis in cardiomyocytes. These results were consistent with previous studied that autophagy promote the survival of cardiomyocytes. Our results reveal that autophagy involves in the inflammatory response of cardiomyocytes and the progression of cardiac diseases by inhibiting pyroptosis.

Pyroptosis, as one of the inflammatory response pathways, is involved in LPS-induced inflammatory response (20). Recent studies have found that pyroptosis plays an important role in cardiovascular disease (23). In addition, pyroptosis has been found to be associated with autophagy (24-28). In our research, we further investigated the role of pyroptosis in inflammatory response of cardiomyocytes and the relationship of pyroptosis and autophagy. By performing western blot and knockdown of LC3B, we draw the conclusion that autophagy and pyroptosis are negatively correlated in cardiomyocytes. These results were consistent with previous studies. For example, hypothermia has been reported to alleviate cerebral ischemic injury by autophagy activation and pyroptosis suppression (29). Prostatic hyperplasia has been reported to resulted by protein peroxiredoxin 3 according to inhibiting autophagy and activating pyroptosis (30).

PARP-1, as a damage repair enzyme, is widely involved in apoptosis-related pathways and has been reported to 

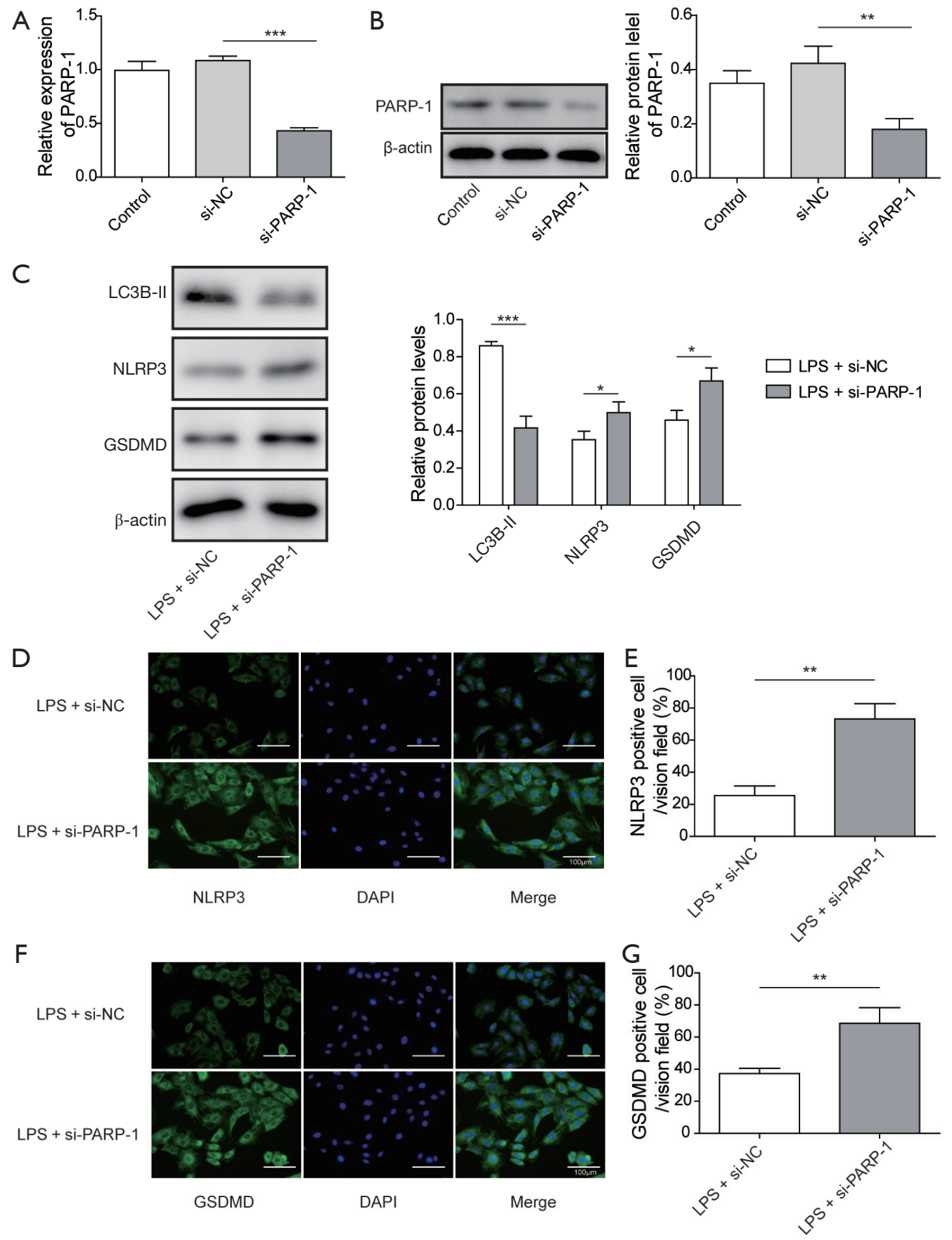

Figure 4 Inhibiting PARP-1 rescued the inhibition of autophagy on LPS-induced pyroptosis of cardiomyocytes. Cardiomyocytes were transfected with si-PARP-1 and treated with LPS of $4 \mu \mathrm{g} / \mathrm{mL}$. (A) PARP-1 mRNA expression was measured by qRT-PCR. (B) Western blots were performed to quantify PARP-1 protein expression. (C) Western blots were performed to determine the expression of LC3B-II, NLRP3 and GSDMD in cardiomyocytes after transfected with si-PARP-1 and treated with $4 \mu \mathrm{g} / \mathrm{mL}$ LPS. (D,E) Expression of NLRP3 in cardiomyocytes were detected by IF staining after transfected with si-PARP-1 and treated with LPS of $4 \mu \mathrm{g} / \mathrm{mL}$. (F,G) Expression of GSDMD in cardiomyocytes were detected by IF staining after transfected with si-PARP-1 and treated with LPS of $4 \mu \mathrm{g} / \mathrm{mL}$. *, P<0.05; **, $\mathrm{P}<0.01$; **, $\mathrm{P}<0.001$. PARP-1, poly ADP-ribosepolmesera-1; LPS, lipopolysaccharide; qRT-PCR, quantitative real-time PCR; IF, immunofluorescence. 
associated with structure and function of heart. Previous studies have shown that PARP-1 plays an important role in neuronal apoptosis and cardiovascular disease $(31,32,37,38)$. In our study, we confirmed that PARP-1 binding to LC3B and regulate the expression of LC3B by performing Co-IP and qRT-PCR. Furthermore, we proved that knockdown of PARP-1 rescued the inhibition of autophagy on LPSinduced pyroptosis of cardiomyocytes with the help of western blot and IF. These results were consistent with previous studies that PARP-1 promotes autophagy in cardiomyocytes by regulating FoxO3a (37) and inhibition attenuates cardiac fibrosis resulted from myocardial infarction via modulating autophagy (38). To sum up, our results revealed that LPS modulates pyroptosis through regulating autophagy via PARP-1 in cardiomyocytes.

According to our study, LPS induces autophagy and pyroptosis of cardiomyocytes and autophagy inhibits LPS induced pyroptosis. We confirmed that PARP-1 binding to LC3B and regulate the expression of LC3B. Finally, we proved that knockdown of PARP-1 rescued the inhibition of autophagy on LPS-induced pyroptosis of cardiomyocytes. To sum up, we draw the conclusion that LPS regulates autophagy via PARP-1 in cardiomyocytes, and PARP-1 plays roles by binding to LC3B. Furthermore, we clarified the role of LPS and PARP-1 regulating autophagy and pyroptosis in the occurrence and development of inflammatory process in cardiomyocytes, which bring new insight for the prevention and treatment of cardiovascular diseases.

\section{Acknowledgments}

Funding: This work was supported by Youth Foundation of Guizhou Provincial People's Hospital (GZSYQN [2018] No. 07), Youth Scientific Research Funding Project of Guizhou Provincial People's Hospital (GZSYQN [2018]), Guizhou Provincial Health Commission project number (gzwkj 2021-102), Guizhou Provincial Traditional Chinese Medicine Project Fund (QZYY-2021-001), Guizhou Provincial Science and Technology Agency Project (Qian Ke He Basic-ZK [2021] General 358), Guizhou Provincial High-level innovative talent training plan (Qian $\mathrm{Ke} \mathrm{He}$ Talent (2016) 4023), and Guizhou Provincial Technology platform and talent team planning project (Qian Ke He Platform Talent (2017) 5405).

\section{Footnote}

Reporting Checklist: The authors have completed the
MDAR reporting checklist. Available at https://dx.doi. org/10.21037/cdt-21-293

Data Sharing Statement: Available at https://dx.doi. org/10.21037/cdt-21-293

Peer Review File: Available at https://dx.doi.org/10.21037/ cdt-21-293

Conflicts of Interest: All authors have completed the ICMJE uniform disclosure form (available at https://dx.doi. org/10.21037/cdt-21-293). The authors have no conflicts of interest to declare.

Ethical Statement: The authors are accountable for all aspects of the work in ensuring that questions related to the accuracy or integrity of any part of the work are appropriately investigated and resolved.

Open Access Statement: This is an Open Access article distributed in accordance with the Creative Commons Attribution-NonCommercial-NoDerivs 4.0 International License (CC BY-NC-ND 4.0), which permits the noncommercial replication and distribution of the article with the strict proviso that no changes or edits are made and the original work is properly cited (including links to both the formal publication through the relevant DOI and the license). See: https://creativecommons.org/licenses/by-nc-nd/4.0/.

\section{References}

1. Klionsky DJ, Emr SD. Autophagy as a regulated pathway of cellular degradation. Science 2000;290:1717-21.

2. Levine B, Kroemer G. Autophagy in the pathogenesis of disease. Cell 2008;132:27-42.

3. Nakatogawa H, Suzuki K, Kamada Y, et al. Dynamics and diversity in autophagy mechanisms: lessons from yeast. Nat Rev Mol Cell Biol 2009;10:458-67.

4. Zheng S, Han F, Shi Y, et al. Single-Prolonged-StressInduced Changes in Autophagy-Related Proteins Beclin-1, LC3, and p62 in the Medial Prefrontal Cortex of Rats with Post-traumatic Stress Disorder. J Mol Neurosci 2017;62:43-54.

5. Schmitz KJ, Ademi C, Bertram S, et al. Prognostic relevance of autophagy-related markers LC3, p62/ sequestosome 1, Beclin-1 and ULK1 in colorectal cancer patients with respect to KRAS mutational status. World J Surg Oncol 2016;14:189. 
6. Pattingre S, Tassa A, Qu X, et al. Bcl-2 antiapoptotic proteins inhibit Beclin 1-dependent autophagy. Cell 2005;122:927-39.

7. Chen $\mathrm{Y}, \mathrm{Li} \mathrm{X}, \mathrm{Wu} \mathrm{X}$, et al. Autophagy-related proteins LC3 and Beclin-1 impact the efficacy of chemoradiation on esophageal squamous cell carcinoma. Pathol Res Pract 2013;209:562-7.

8. Hamurcu Z, Delibaşı N, Geçene S, et al. Targeting LC3 and Beclin-1 autophagy genes suppresses proliferation, survival, migration and invasion by inhibition of Cyclin-D1 and uPAR/Integrin $\beta 1 /$ Src signaling in triple negative breast cancer cells. J Cancer Res Clin Oncol 2018;144:415-30.

9. Adams O, Dislich B, Berezowska S, et al. Prognostic relevance of autophagy markers LC3B and p62 in esophageal adenocarcinomas. Oncotarget 2016;7:39241-55.

10. Hamurcu Z, Delibaşı N, Nalbantoglu U, et al. FOXM1 plays a role in autophagy by transcriptionally regulating Beclin-1 and LC3 genes in human triple-negative breast cancer cells. J Mol Med (Berl) 2019;97:491-508.

11. Masuda GO, Yashiro M, Kitayama K, et al. Clinicopathological Correlations of Autophagy-related Proteins LC3, Beclin 1 and p62 in Gastric Cancer. Anticancer Res 2016;36:129-36.

12. Oh SY, Choi SJ, Kim KH, et al. Autophagy-related proteins, LC3 and Beclin-1, in placentas from pregnancies complicated by preeclampsia. Reprod Sci 2008;15:912-20.

13. Chen L, Wang FY, Zeng ZY, et al. MicroRNA-199a acts as a potential suppressor of cardiomyocyte autophagy through targeting Hspa5. Oncotarget 2017;8:63825-34.

14. Criollo A, Altamirano F, Pedrozo Z, et al. Polycystin-2dependent control of cardiomyocyte autophagy. J Mol Cell Cardiol 2018;118:110-21.

15. Li R, Wang X, Wu S, et al. Irisin ameliorates angiotensin II-induced cardiomyocyte apoptosis through autophagy. J Cell Physiol 2019;234:17578-88.

16. Zhang E, Zhao X, Zhang L, et al. Minocycline promotes cardiomyocyte mitochondrial autophagy and cardiomyocyte autophagy to prevent sepsis-induced cardiac dysfunction by Akt/mTOR signaling. Apoptosis 2019;24:369-81.

17. Su B, Wang XT, Sun YH, et al. Ischemia/hypoxia inhibits cardiomyocyte autophagy and promotes apoptosis via the Egr-1/Bim/Beclin-1 pathway. J Geriatr Cardiol 2020;17:284-93.

18. Zhao D, Gao Y, Wang W, et al. PKD deletion promotes autophagy and inhibits hypertrophy in cardiomyocyte. Exp Cell Res 2020;386:111742.
19. Gao G, Chen W, Yan M, et al. Rapamycin regulates the balance between cardiomyocyte apoptosis and autophagy in chronic heart failure by inhibiting mTOR signaling. Int J Mol Med 2020;45:195-209.

20. Kesavardhana S, Malireddi RKS, Kanneganti TD. Caspases in Cell Death, Inflammation, and Pyroptosis. Annu Rev Immunol 2020;38:567-95.

21. Lacey CA, Mitchell WJ, Dadelahi AS, et al. Caspase-1 and Caspase-11 Mediate Pyroptosis, Inflammation, and Control of Brucella Joint Infection. Infect Immun 2018;86:e00361-18.

22. Ma Y, Jiang J, Gao Y, et al. Research progress of the relationship between pyroptosis and disease. Am J Transl Res 2018;10:2213-9.

23. Wu J, Lin S, Wan B, et al. Pyroptosis in Liver Disease: New Insights into Disease Mechanisms. Aging Dis 2019;10:1094-108.

24. Byrne BG, Dubuisson JF, Joshi AD, et al. Inflammasome components coordinate autophagy and pyroptosis as macrophage responses to infection. mBio 2013;4:e00620-12.

25. Kim JY, Paton JC, Briles DE, et al. Streptococcus pneumoniae induces pyroptosis through the regulation of autophagy in murine microglia. Oncotarget 2015;6:44161-78.

26. Kong Y, Feng Z, Chen A, et al. The Natural Flavonoid Galangin Elicits Apoptosis, Pyroptosis, and Autophagy in Glioblastoma. Front Oncol 2019;9:942.

27. Ma SM, Mao Q, Yi L, et al. Apoptosis, Autophagy, and Pyroptosis: Immune Escape Strategies for Persistent Infection and Pathogenesis of Classical Swine Fever Virus. Pathogens 2019;8:239.

28. Wei Q, Zhu R, Zhu J, et al. E2-Induced Activation of the NLRP3 Inflammasome Triggers Pyroptosis and Inhibits Autophagy in HCC Cells. Oncol Res 2019;27:827-34.

29. Tu Y, Guo C, Song F, et al. Mild hypothermia alleviates diabetes aggravated cerebral ischemic injury via activating autophagy and inhibiting pyroptosis. Brain Res Bull 2019;150:1-12.

30. Jiang MY, Han ZD, Li W, et al. Mitochondrion-associated protein peroxiredoxin 3 promotes benign prostatic hyperplasia through autophagy suppression and pyroptosis activation. Oncotarget 2017;8:80295-302.

31. Rodríguez-Vargas JM, Oliver-Pozo FJ, Dantzer F. PARP1 and Poly(ADP-ribosyl)ation Signaling during Autophagy in Response to Nutrient Deprivation. Oxid Med Cell Longev 2019;2019:2641712.

32. Kong Q, Li Y, Liang Q, et al. SIRT6-PARP1 is involved 
in HMGB1 polyADP-ribosylation and acetylation and promotes chemotherapy-induced autophagy in leukemia. Cancer Biol Ther 2020;21:320-31.

33. Huang D, Kim DS, Kraus WL. Specific Binding of snoRNAs to PARP-1 Promotes NAD+-Dependent Catalytic Activation. Biochemistry 2020;59:1559-64.

34. Kadam A, Jubin T, Roychowdhury R, et al. Role of PARP1 in mitochondrial homeostasis. Biochim Biophys Acta Gen Subj 2020;1864:129669.

35. Prasad R, Horton JK, Wilson SH. Requirements for PARP-1 covalent crosslinking to DNA (PARP-1 DPC). DNA Repair (Amst) 2020;90:102850.

Cite this article as: He YF, Huang J, Qian Y, Liu DB, Liu QF. Lipopolysaccharide induces pyroptosis through regulation of autophagy in cardiomyocytes. Cardiovasc Diagn Ther 2021;11(5):1025-1035. doi: 10.21037/cdt-21-293
36. Siavashpour A, Khalvati B, Azarpira N, et al. Poly (ADPRibose) polymerase-1 (PARP-1) overactivity plays a pathogenic role in bile acids-induced nephrotoxicity in cholestatic rats. Toxicol Lett 2020. [Epub ahead of print]. doi: 10.1016/j.toxlet.2020.05.012.

37. Wang $\mathrm{C}, \mathrm{Xu} \mathrm{W}$, Zhang Y, et al. PARP1 promote autophagy in cardiomyocytes via modulating FoxO3a transcription. Cell Death Dis 2018;9:1047.

38. Wang H, Yang X, Yang Q, et al. PARP-1 inhibition attenuates cardiac fibrosis induced by myocardial infarction through regulating autophagy. Biochem Biophys Res Commun 2018;503:1625-32. 


\section{Supplementary}
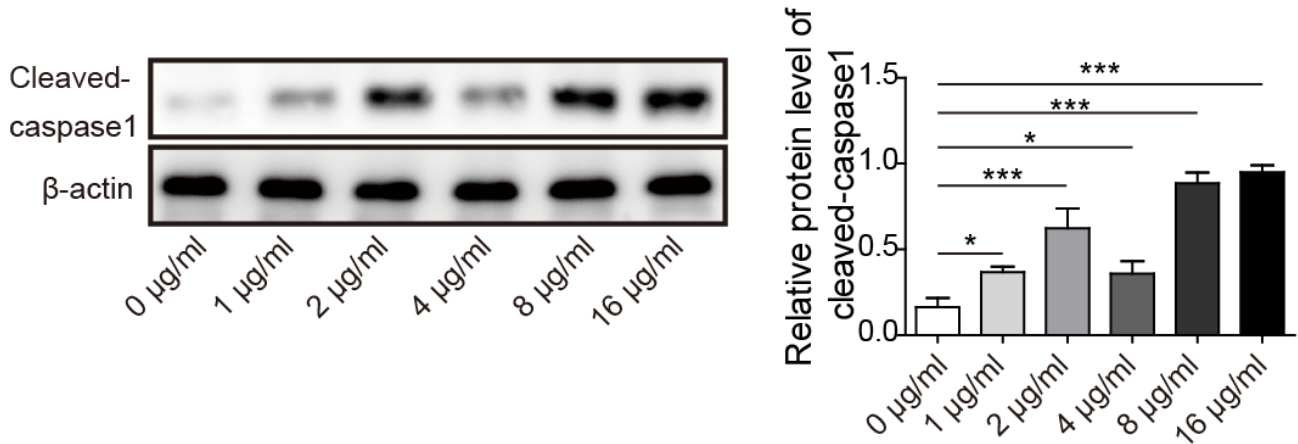

Figure S1 Expression of cleaved caspase-1 was the lowest in cardiomyocytes treated by LPS of $4 \mu \mathrm{g} / \mathrm{mL}$. Expression of cleaved caspase-1 in cardiomyocytes treated by different concentration of LPS. *, $\mathrm{P}<0.05$; ${ }^{* * *}, \mathrm{P}<0.001$. LPS, lipopolysaccharide.
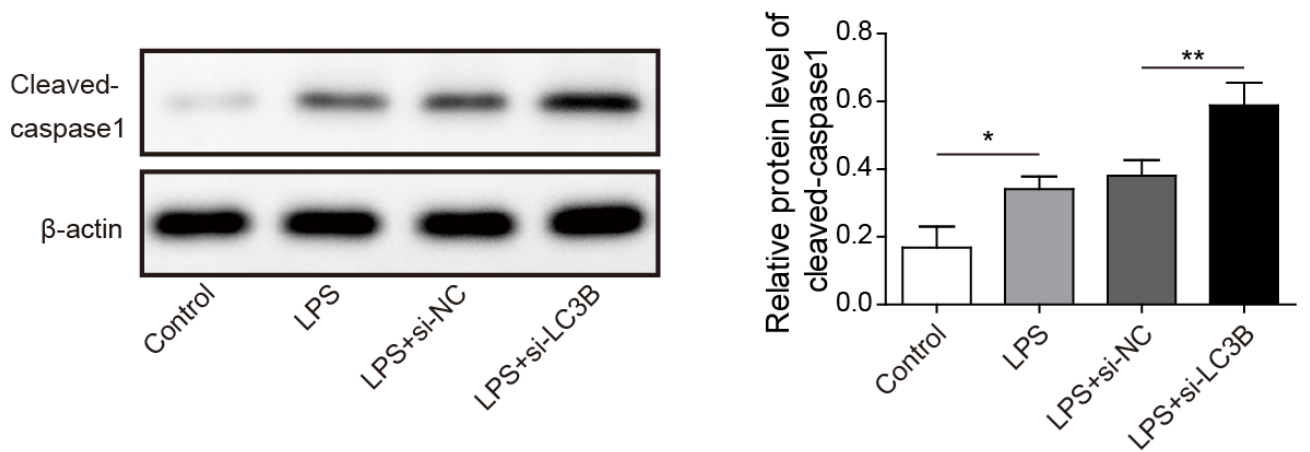

Figure S2 Inhibition of autophagy enhanced the level of pyroptosis. Expression of cleaved caspase-1 in cardiomyocytes after treated with LPS and transfected with si-LC3B. *, $\mathrm{P}<0.05$; **, $\mathrm{P}<0.01$. LPS, lipopolysaccharide.
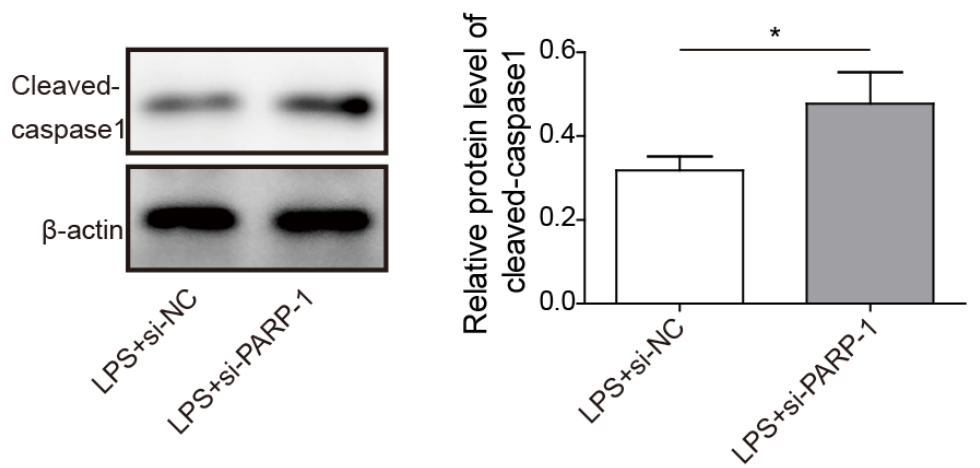

Figure S3 Knockdown of PARP-1 enhanced the expression of cleaved caspase-1. Expression of cleaved caspase-1 in cardiomyocytes after treated with LPS and transfected with si-PARP-1. *, P<0.05. LPS, lipopolysaccharide; PARP-1, poly ADP-ribosepolmesera-1. 